\title{
Damage Avoidance Design Steel Beam-Column Moment Connection Using High-Force-To-Volume Dissipators
}

\author{
Thomas J. Mander; Geoffrey W. Rodgers; J. Geoffrey Chase; John B. Mander; and \\ Gregory A. MacRae
}

\begin{abstract}
Existing welded steel moment frames are designed to tolerate substantial yielding and plastic rotation under earthquake loads. This sacrificial design approach can lead to permanent, and often irreparable damage when interstory drifts exceed $2 \%$. The experimental seismic performance of a $50 \%$ full-scale damage avoidance designed structural steel beam-column connection is presented. The beamcolumn joint region consists of a top flange-hung beam connected to the column by an angle bracket. High-force-to-volume (HF2V) devices are attached from the column to the beam to provide joint rigidity and energy dissipation as the joint opens and closes. The HF2 V devices are connected either below the beam flange or concealed above the beam's lower flange. Reversed cyclic lateral load tests are conducted with drift amplitudes up to $4 \%$. No damage is observed in the principal beam and column structural elements. The need for stiff device connections to achieve optimal device performance is demonstrated, and potential design solutions presented. Stable hysteresis and repeatable energy dissipation for a large number of cycles up to the $4 \%$ drift level is observed. It is concluded that superior and repeatable energy dissipation without damage can be achieved for every dynamic motion cycle, in contrast to conventional sacrificially designed welded moment frame connections.
\end{abstract}

UCRR http://ir.canterbury.ac.nz/handle/10092/3580

CE Database subject headings: Steel structures; Damage; Energy dissipation; Beam columns; Connections; Frames.

\section{Introduction}

Occupant safety and use of steel frame buildings following major earthquakes is often limited by damage to the structural frame system. Most of this damage is restricted to the plastic hinge zones at beam ends or the panel zone within steel beam-column joints. As evidenced by the extensive damage to steel structures following the 1994 Northridge earthquake, repair costs and downtime can be substantial to owners and users of such structures, creating a significant long-term social and economic impact.

Presently, steel connections are designed using sacrificial yielding at beam ends to dissipate dynamic response energy. Plastic hinges form at the ends of beams, causing permanent damage and the possibility of failure under extreme interstory drifts due to flange buckling or weld fracture. The associated damage can be difficult, time consuming, and expensive to repair. It is thus desirable to have damage-free connections, eliminating expensive

\footnotetext{
${ }^{1}$ Graduate Assistant Researcher, Zachry Dept. of Civil Engineering, Texas A\&M Univ., College Station, TX 77840.

${ }^{2} \mathrm{Ph}$.D. Candidate, Dept. of Mechanical Engineering, Univ. of Canterbury, Christchurch 8140, New Zealand (corresponding author).

${ }^{3}$ Professor, Dept. of Mechanical Engineering, Univ. of Canterbury, Christchurch 8140, New Zealand.

${ }^{4}$ Zachry Professor, Design and Construction Integration, Zachry Dept. of Civil Engineering, Texas A\&M Univ., College Station, TX 77840.

${ }^{5}$ Associate Professor, Dept. of Civil Engineering, Univ. of Canterbury, Christchurch 8140, New Zealand.
}

repairs and enabling businesses to continue without causing further financial loss to the owner or tenants.

Compact high-force-to-volume (HF2V) energy dissipating de- vices using a bulged shaft in a prestressed lead cylinder have been developed and experimentally characterized (Rodgers et al.

2007). These devices are capable of fitting within standard struc- tural connections. They are also inexpensive to manufacture, po- tentially making them economically feasible for widespread use in large buildings. The use of supplementary HF2V devices fitted directly into beam-column connections provides a damage avoid- ance design (DAD) structural connection. The devices use lead

because of its unique rheological properties, low recrystallization temperature to give consistent force levels on successive cycles, and the ability for lead to creep over time, giving the device the ability to allow residual compression forces to dissipate through creep effects. With these devices, the same or a greater amount of energy can be dissipated on successive cycles and without caus- ing permanent damage, unlike conventional steel connections or sacrificial dissipators (Solberg et al. 2008).

Previous research has also investigated the concept of damage- free steel structures. The inclusion of posttensioned steel tendons provides self-centering ability to the joint (Christopoulos et al.

2002; Ricles et al. 2001; Pekcan et al. 2000), with energy dissi- pation typically provided by either friction, viscous, or yielding steel devices (Christopoulos et al. 2008; Garlock et al. 2005). The systems that utilize posttensioned tendons have the advantage of joint self-centering. However, these systems also have the limita- tion that they are dilating frame systems, which must be accom- modated by gap opening or tearing of the flooring system during earthquake response cycles, as discussed in Garlock et al. (2007).

This paper presents the results of an experimental investiga- tion on the seismic performance of DAD structural steel beam- column connections using these HF2V devices. Instead of 
conventional welding, a bolted top angle and a HF2V device form the moment connection. Two different experimental setups are investigated. Results are reported and discussed for repeated reversed cyclic tests up to $4 \%$ drift amplitudes.

For mitigating seismic response, Robinson and Greenbank $(1975,1976)$ first proposed a class of damping devices that used the principle of extrusion of the working material-lead. These so-called lead extrusion damping (LED) devices were quite large and developed relatively modest forces for their size. They were designed to absorb structural energy during an earthquake in a controlled manner, with repeatable behavior. A typical application was envisioned as base isolation systems, which were not volumetrically constrained. Large LED devices have been deployed in bridges and buildings in New Zealand (Skinner et al. 1993). A summary of the state-of-the-practice for this type of device, as developed from this early work, is given by Cousins and Porritt (1993).

In contrast to the very large dampers previously deployed, the present research is focused on developing such behavior, but using devices with a HF2V ratio. Such devices have been developed successfully by Rodgers et al. (2007), optimizing the dimensions of the bulged damper shaft and cylinder. Previous near fullscale precast concrete jointed beam-to-column connection experiments have focused on using these devices either externally mounted (Solberg 2007) or embedded within concrete at the beam end and connected through the column (Solberg et al. 2007). While the externally mounted design provides an easily maintainable system, the concealed (embedded) design provides an architecturally pleasing finish; both solutions provide a near-rigid connection. Both of these previous studies on precast concrete frame systems have demonstrated proof-of-concept evidence for a reliable form of DAD by adopting these HF2V devices as the principal means of energy dissipation.

The present work focuses on implementing the HF2V devices in a similar fashion for steel moment frames. The devices are either mounted for maximum efficiency below the bottom flange of the beam adjacent to the column face, or connected to the beam web just above the bottom flange so they are concealed in a building's roof space.

To summarize, the purpose of this research is to investigate the viability of using HF2V devices in rigid structural steel beam-tocolumn connections as part of implementing DAD principles into structural steel frame systems. This approach has the advantage that plastic hinging and hence damage can be avoided during seismic response; this is in contrast to current design practice. Present codes generally specify rigid welded connections that are expected to sustain considerable sacrificial damage dissipating energy in earthquakes. Such damage is likely to be irreparable because beam flanges can become buckled, fatigued, or fractured.

\section{Concept Development}

The loading conditions and the implementation of the HF2V device into a steel beam-column joint for the experimental tests imitate that of a typical steel structure. The basis for the experimental setup is provided in Fig. 1. Based on typical prototype steel building dimensions [Fig. 1(a)] an approximate $50 \%$ scale physical model [Fig. 1(b)] was developed.

Within a moment resisting connection, the resistance force provided by the device, $F_{d}$, leads to a joint moment defined
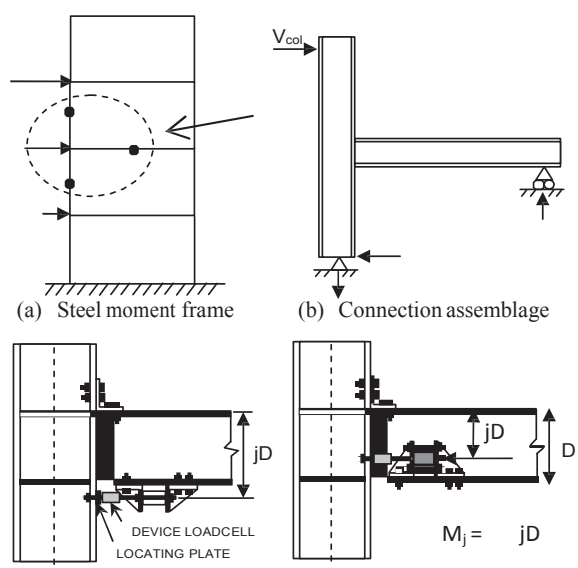

(c) HF2V device mounted below beam $(\mathrm{j}>1)$

b) Connection assemblage

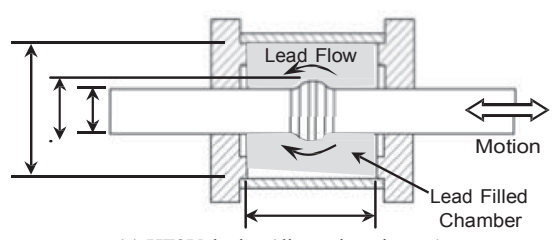

(e) HF2V device (dimensions in $\mathrm{mm}$ )

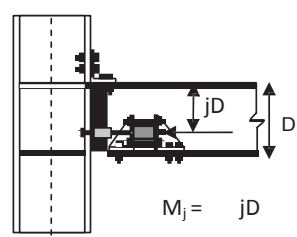

(d) Concealed mounting of

HF2V devices $(j<1)$

Fig. . Steel moment frames fitted with HF2V devices

$$
M_{j}=F_{d} j D
$$

where $F_{d}=$ damper force during plastic flow; $D=$ overall depth of the steel girder (beam); and $j=$ proportion of the beam depth be- tween the upper (flange) and lower (device) forces, as shown in

Figs. 1(c and $d$ ).

The HF2V device is compact, similar in size to a soft drink can, allowing it to fit directly beneath [Fig. 1(c)] or concealed within the beam at the beam-to-column connection [Fig. 1(d)].

The same devices used previously by Solberg et al. (2007) are used in this work. They have a stroke of $20 \mathrm{~mm}$ and a shaft yield force of $180 \mathrm{kN}$. The dampers were modified for the testing of the device below the bottom flange as the configuration requires a

longer device stroke. The cylinder of the device was lengthened, allowing increased travel of the damper shaft, providing a stroke of $32 \mathrm{~mm}$.

Fig. 1(e) presents a cross-sectional view of a HF2V device used in this research. Such devices provide a resistive force from the bulged steel shaft passing through the lead-filled chamber. In contrast to the original lead extrusion devices of Robinson and Greenbank $(1975,1976)$, the new generation HF2V devices have their lead prestressed within the cylinder. Once the initial pre- stress effects are overcome the lead plastically deforms and pro- vides a large drag force in the wake of the bulge. This dissipates considerable energy through shearing and general internal plastic

deformation of the lead.

When appropriately implemented into a typical steel beam- column joint, under lateral frame drift hysteretic energy is dissi- pated though the HF2V device. To activate movement in the dissipator the connection needs to be able to rotate. A bolted connection, rather than a typical moment resisting welded con- nection, permits the required rotation to take place without induc- ing damage (via yielding) to the structural beam and column elements. A bolted connection should suffice for the purpose of

connecting the beam, column, and HF2V parts obviating the need to undertake costly on-site welding. 


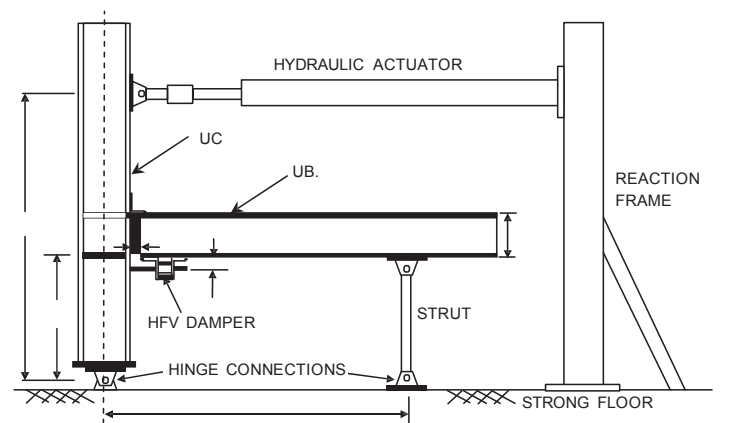

(a) Specimen setup for lateral load tests on strong-floor
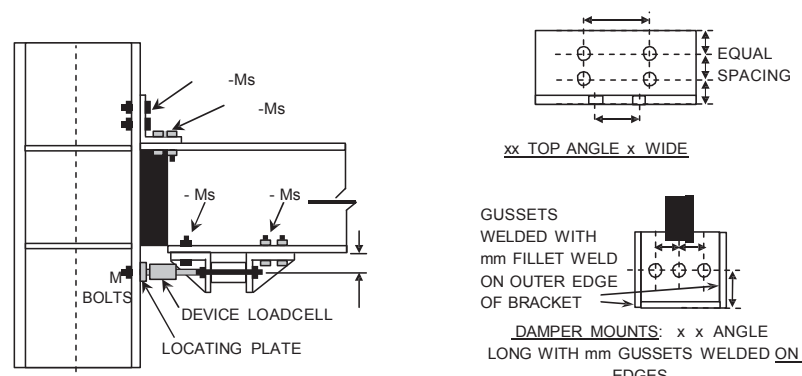

xx TOP ANGLE $x$ WIDE

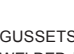

WELDED WITH

mm FILLET WELD

Mm FILLET WELD
ON OUTER EDGE
OF BRACKET

DAMPER MOUNTS $X \times$ ANGE

DAMPER MOUNTS: $\times$ × ANGLE
LONG WITH mm GUSSETS WELDED ON EDGES

(b) Details of HF2V device mounted below bottom flange of beam ( $\mathrm{jD}=412)$
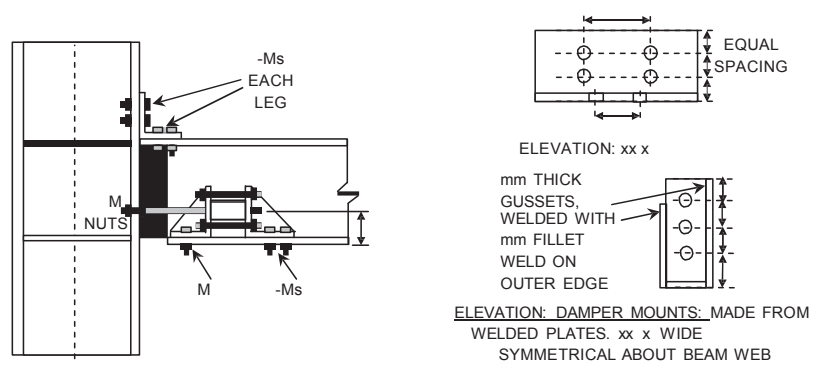

(c) Details of concealed mounting of HF2V fitted on both sides of web (jD=232)

Fig. . Specimen details for experimental study (dimensions in millimeters)

\section{Experimental Apparatus and Study}

Fig. 2 presents the experimental setup of the exterior beam-tocolumn structural steel connection tested in this research. An angle was bolted to the top flange of the 360UB44.7 beam, which in turn was bolted to the $310 \mathrm{UC} 158$ column with M20 bolts, as detailed in Fig. 2(b). Grade 300 hot rolled structural steel was specified for both the beam and column members.

Quasi-static loading was used to test the beam-column joint specimen with the HF2V devices [Fig. 2(a)], up to a maximum peak drift of $4 \%$. The theoretical capacity of the lateral load applied to the column to dissipate energy is $28 \mathrm{kN}$ and $38 \mathrm{kN}$ for a single device mounted below the bottom beam flange [Fig. 2(b)], and two devices mounted above the bottom flange [Fig. 2(c)], respectively. The devices are intended to provide all energy dissipation while the top angle essentially acts as a hinge.

An important design feature is the provision of a $30-\mathrm{mm}$ movement gap between the column face and bottom beam flange, as shown in Fig. 2. The beam was cut back at an angle of approximately $5^{\circ}$ to prevent the bottom flange from damaging the column under joint closing up to $4 \%$ drift, permitting free-hinged motion. Lateral load and displacement of the column were provided by a $100-\mathrm{kN}$ capacity hydraulic actuator with a $500 \mathrm{~mm}$

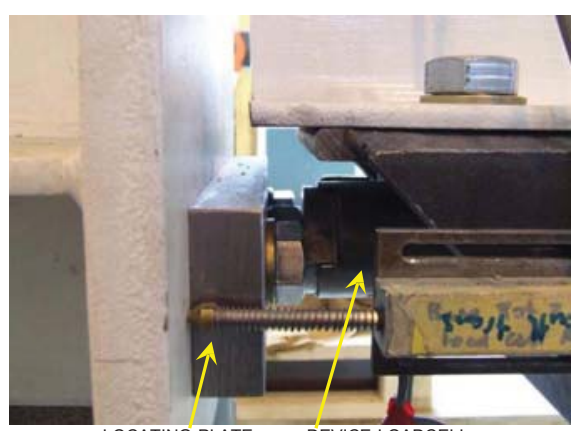

LOCATING PLATE DEVICE LOADCELL
(a) Detail of beneath-flange mounted HF2V device

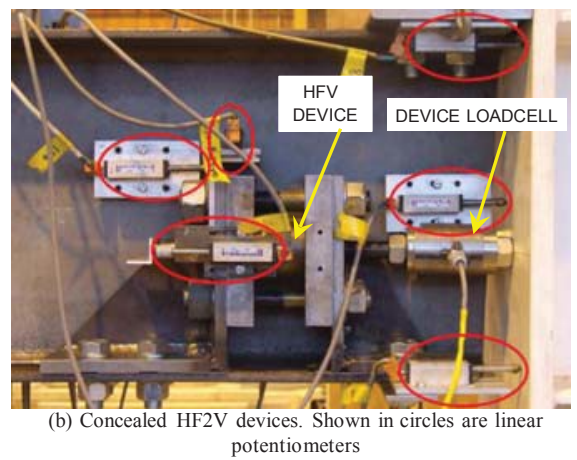

Fig. . Photographs of the experimental specimens showing the $\mathrm{HF} 2 \mathrm{~V}$ devices in the joint zone

above the hinge connection at the base of the column that was in turn connected to the laboratory strong floor. An end plate was welded to the base of the column to attach the column to the base hinge.

Column top displacement was measured with an external string (rotary) potentiometer. Load cells connected to the damper shafts measured the forces in the HF2V devices. A potentiometer was screwed into the end of the shaft to measure the displacement of the shaft in the cylinder. Other linear potentiometers were placed to capture potential losses of stiffness and undesired move- ment in the subassembly, particularly due to device bracket com- pliance. Fig. 3 shows the instrumentation used for the damper located above the bottom flange. Linear potentiometers measured horizontal and vertical displacement in the HF2V devices mounts, the top angle, and base of the beam flange relative to column face.

\section{HFV Device Location}

Placing the device beneath the bottom flange $j>1$ ) provides the greatest lever arm between the device and top connection. This arrangement is preferred if the connection cost is to be mini- mized, as the damper force is minimal. However, in a regular office building, unless this can be made an architectural feature, such a placement beneath the beam may affect the wall and ceil- ing placement. Placing the device between the beam flanges $j$

1) avoids this issue. For both arrangements, an important re-

quirement for device performance is a nonslip condition between

stroke. The actuator was attached to the top of the column $2.0 \mathrm{~m}$ 
the damper and the beam flange. Any slip present will reduce the force provided to the overall joint, reducing the overall dissipa- tion and reducing the efficiency of the dampers. Locating the damper below the bottom flange enables an increased number of bolts to be used (bolting on either side of the web for one device rather than a single side for the device above the bottom flange).

When the damper was mounted below the beam flange, a total of 


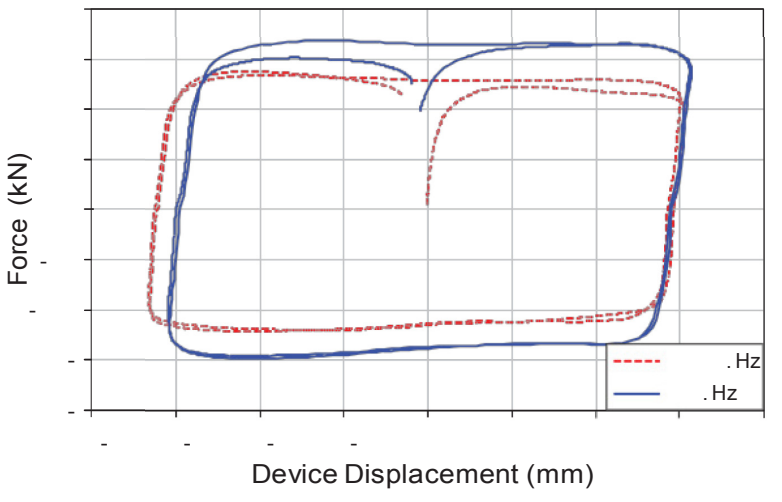

(a) HF2V device results for two cyclic test frequencies

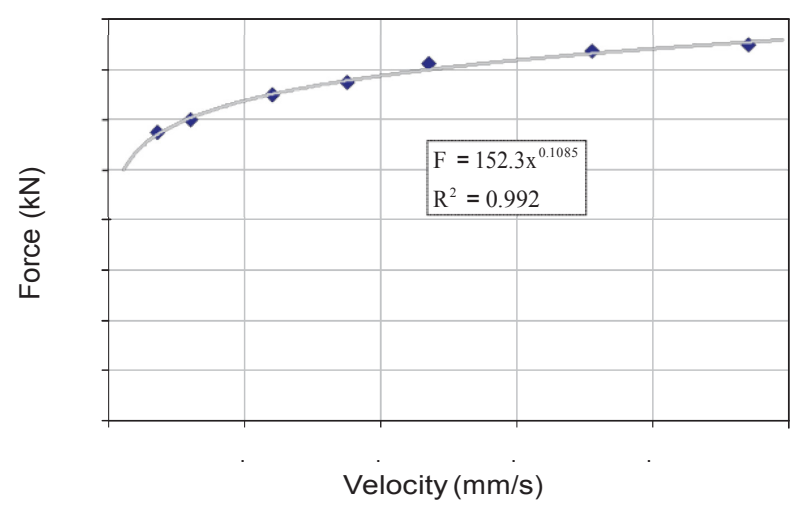

(b) Empirical velocity dependent power law

Fig. . HF2V device used in the beam-column joint experiment showing velocity-dependent performance

six Grade $8.8 \mathrm{M} 24$ bolts were used and proof loaded to ensure a nonslip condition, as shown in Fig. 2(b). In contrast to this, only one-half of the number of bolts per damper were used for the case when the two dampers were mounted above the flange, as shown in Fig. 2(c).

Positioning the damper at the beam and column centerline complicates the connection of the damper shaft to the column, as the shaft coincides with the column web. The damper cannot be bolted directly through the column flange, therefore a locating plate that was drilled and tapped at its midpoint to allow attachment of the device loadcell was designed. This plate was bolted through the column face by two M20 bolts, each located at 50 $\mathrm{mm}$ from the column centerline, allowing for clearance of the bolt head from the column web and root radius, as shown in Fig. 3(a).

The damper configuration and mounting detail enabled a nonslip condition using an entirely bolted connection, an important economic consideration as it eliminates the need for any on-site welding, a specialized trade that usually requires inspection. Although the top angle provides a shear connection, this damper configuration also enables a shear connection to be made to the beam web if extra reinforcement is required. This shear connection can be slotted in the horizontal direction, enabling effective shear transfer without preventing joint rotation.

\section{Device Characterization}

Cyclic tests were conducted to obtain the nonlinear velocity dependence of the HF2V devices. The tests were carried out at frequencies from 0.0015 to $0.02 \mathrm{~Hz}$. The upper test frequency was limited to ensure the damper shaft did not yield at the forces produced by the devices. Results of these tests are presented in Fig. 4. These results match the device characterization hysteresis obtained by Solberg et al. (2007) and the original device calibrations of Rodgers et al. (2007). The essentially square hysteresis loops and consistent force capacity indicate the potentially large energy dissipation that can be obtained [Fig. 4(a)].

From the tests conducted the maximum average force was obtained for each frequency. The maximum force versus maximum velocity trend presented in Fig. 4(b) shows no obvious outliers. The devices are almost elastic perfectly plastic in their behavior, but with a mild velocity sensitivity, with the yield force related to the velocity. The HF2V damper can thus be modeled as where the damper force $F_{d}$ ) is defined (Pekcan et al. 1999)

$$
F_{d}=C \dot{x}
$$

where $C=$ a constant depending on the device geometry and working material; $\dot{x}=$ the velocity of the damper shaft; and $=$ the velocity exponent. For the dampers used in the present investigation it was found from tests that $C=152$ and $=0.11$ (note: $\dot{x}$ is in $\mathrm{mm} / \mathrm{s}$ and $F_{d}$ is in $\mathrm{kN}$ units). The larger forces in the hysteresis loops of Fig. 4(a) clearly indicate the consistent veloc-

ity dependence seen in this curve.

\section{Experimental Behavior}

Quasi-static lateral loading were applied to the structural steel beam-to-column joint subassemblage at drift amplitudes of 0.25 , $0.5,1,2$, and $3 \%$ and four cycles at $4 \%$. Two fully reversed cycles were undertaken (at each drift amplitude) to demonstrate the abil- ity of the joint to undergo large drifts without any stiffness or strength degradation, which is common in other structural mate- rials such as RC and welded steel connections. The resulting ex- perimentally observed lateral load versus drift performance is presented in Fig. 5 for all cycles. It is therefore noted in Fig. 5 that the results are seen to be so consistent that for large sections of the hysteresis loop it is not immediately apparent that multiple cycles have been performed, as the lines from successive cycles are almost indistinguishable. In addition, subsequent smaller drift cycles in the second cycle of testing shared the same level of dissipation, which would not occur with sacrificial fuse ap-

proaches to this type of DAD connection (Solberg et al. 2008).

Based on the mechanics of the connections, Figs. 5( $a$ and $b)$ plot the theoretical lateral strength capacities of $28 \mathrm{kN}$ and $36 \mathrm{kN}$, for the cases of one damper below the flange and two dampers above the flange, respectively. Note that because of the mild ve-

locity dependence, the observed strength tends to exceed this limit at high drifts when the cyclic testing rate correspondingly increased. This effect is shown in Figs. 5(c and d), where the in-joint measured damper force is plotted against the "in service" device movement. The slight increase in force for larger drifts is evident and due to a test procedure that moved the column to each 


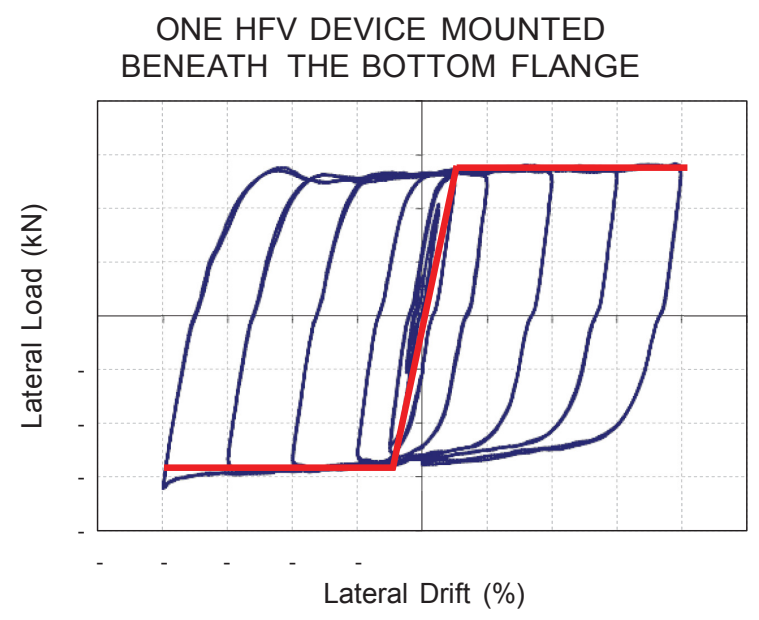

(a) Force-drift response for one HF2V device

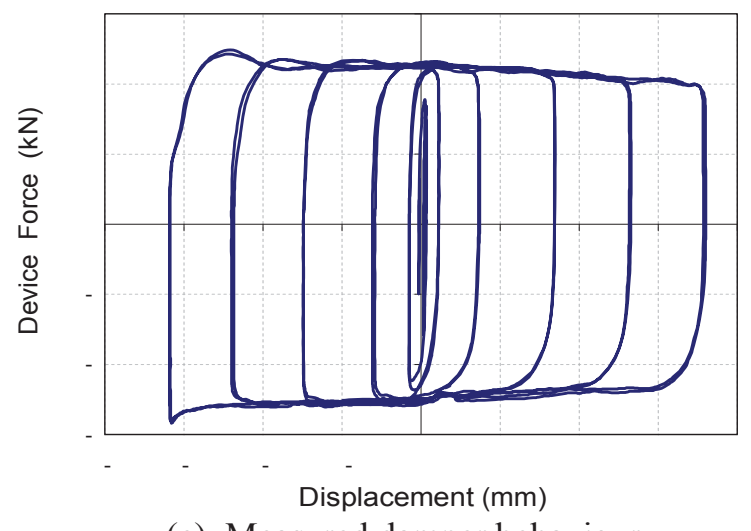

(c) Measured damper behaviour

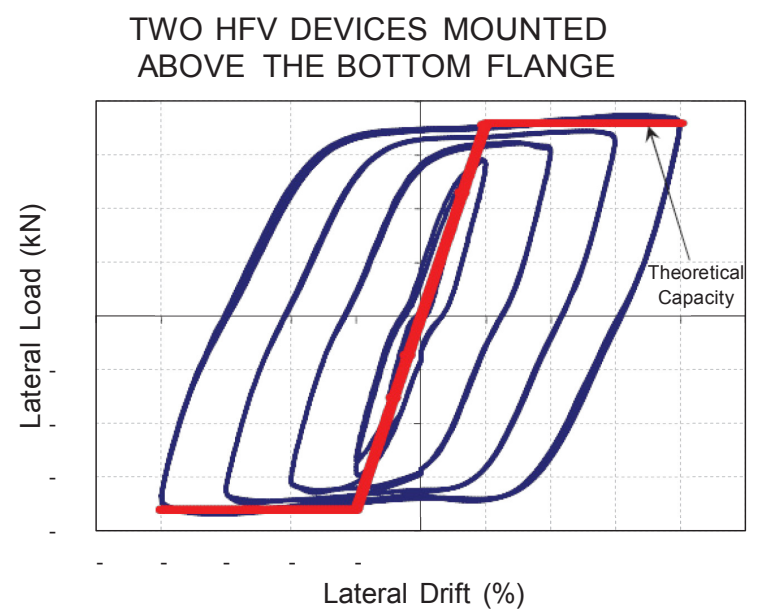

(b) Force-drift response for two HF2V devices

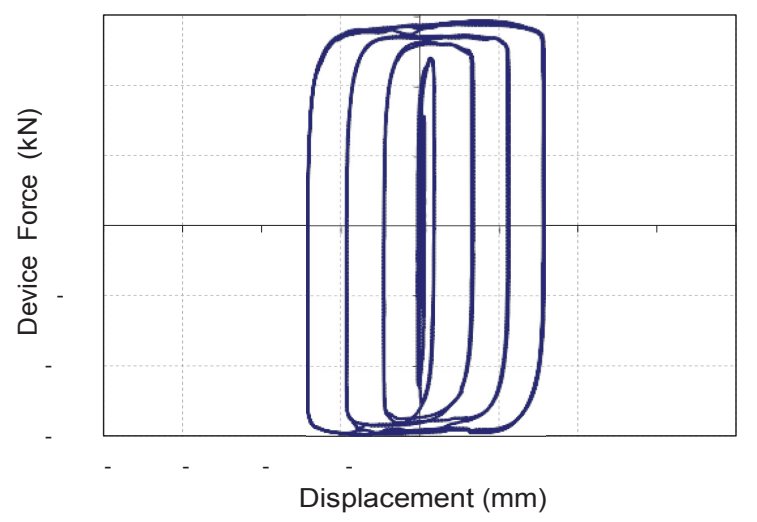

(d) Behaviour of north-side HF2V device

Fig. . Results for the two main experiments: (a),(c) and (b),(d) are shown for fully reversed loading cycles of $0.25,0.5,1.0,2.0,3.0$, and $4.0 \%$ drift. The theoretical capacity is also shown in panels (a) and (b).

\section{Discussion of Results for Damper Mounted below Beam}

The results shown in Fig. 5(a) indicate that the response exhibits some bilinear characteristics. As the overall hysteresis is directly related to the damper performance, it was expected that the inservice hysteretic response of the damper was bilinear as illustrated in Fig. 5(c). The reason for this behavior is not clear, although it may be attributable to the inherent bending stresses applied to the shaft as the bottom of the beam moves in an arc about the top-flange edge. Such action may induce additional friction and binding where the shaft contacts the endcaps. This phenomenon could be eliminated in future work by the use of a clevis design at each location point of the damper, eliminating any bending stresses and ensure that any force in the device is purely axial.

When comparing the overall hysteresis of a bottom mounted device to two devices mounted above the bottom flange it is evident that the single configuration has greater initial stiffness, but lower peak force, as shown in Figs. 5( $a$ and b). These observations can be easily explained by the distance, $j$, of the dampers from the pivoting top-flange edge. The single damper is mounted below the bottom flange, such that $j=1.17$, giving a distance from the top-flange connection of $j D=412 \mathrm{~mm}$, resulting in decreased overall resisting moment being applied to the joint.

Moreover, the theoretical prediction of the yield force for the subassembly is $28 \mathrm{kN}$, very close to the average yield force ob- of the prediction, an important aspect for assessing overall struc- tural performance through capacity design methods. The alterna- tive configuration has two dampers located at $j=0.66$, giving a distance from the top flange of $j D=232 \mathrm{~mm}$ below the top-flange edge. By considering both the force imparted into the subassem- bly and the corresponding lever arm provided by the damper ec- centricity, $j D$, then it is evident that the overall joint moment is reduced. The use of one damper occurs while increasing the lever arm, but the lever arm is less than doubled, so the overall joint

moment is reduced.

\section{Discussion of Results for Two Dampers above Beam Flange}

An important consideration is that the hysteresis loops shown

Figs. 5( $a$ and $b)$ represent an entirely bolted connection for the single device and a bolted-welded connection for the two devices above the bottom flange. This outcome is significant, as better overall hysteretic behavior can be obtained from more economi- cal connection detailing, provided sufficient allowance is given to

allow a nonslip condition on the bolted interfaces.

A larger peak force is easily achieved by incorporating a damper with higher yield force, with experimentally proven devices having been constructed with yield forces up to and exceedserved in the experimental results, clearly showing the accuracy 
ing $350 \mathrm{kN}$, compared with the nominal $120 \mathrm{kN}$ of the devices used for this joint (Rodgers et al. 2007). Therefore, the selection 
of an upper bound of the device force is dependent upon the strength of the structural system itself, and its ability to maintain a nonslip condition rather than the ability to build large enough devices. Furthermore, the use of larger device forces and larger diameter shafts would see the initial stiffness of the subassembly increase significantly, providing a stiffer joint while still maintaining a damage-free inelastic response.

For two devices mounted above the bottom flange the experimental setup has an experimental yield drift of approximately $1 \%$. This result is higher than the expected $0.5 \%$ elastic drift expected based on column, beam, strut, and HF2V device shaft elastic deformation. This increase in elastic motion is likely due to the additional flexibility of the damper (angle) mounts and the inseries load cells connected to the HF2V devices. Instrumentation measured negligible movement vertically or horizontally in the top angle, confirming the rigid hinged nature assumed. Fig. 5(b) shows that the stiffness at low force levels in Quadrants 2 and 4 are somewhat less than in Quadrants 1 and 3. This difference is attributed to the rockinglike flexibility introduced into the system by the HF2V device mounting brackets. This conclusion is supported by the plot in Fig. 5(d) where the device itself exhibits approximately a rigid perfectly plastic performance. Hence, the difference from the device to the total assembly is due to elastic compliance in the device connections.

For the case of two dampers mounted above the flange at $0.5 \%$ drift amplitude there was little movement (approximately 2 $\mathrm{mm}$ ) in the device. This small motion resulted in negligible energy dissipation, as seen in Fig. 5(b). As the lateral load increased the bulge began to move more significantly and more energy was dissipated. Virtually all energy dissipated can be attributed to the device, as there was no yielding of the principal structural elements in the experimental setup.

It should be noted that there was a slightly larger displacement in the device when the beam-column gap opened, compared to when it was closing. Noticeable elongation was observed in the bolts connecting the damper shaft to the column face when the joint was fully open. This elongation is a contributing factor to this difference, as well as the change in mechanism of the top angle bracket. More specifically, when the gap opens the angle closes, putting it into compression. This motion provides more resistance than when in tension, as plastic hinges form below the bolt rows and base of the angle (Mander et al. 1994).

The physical gap of $30 \mathrm{~mm}$ between the column face and bottom beam flange provided adequate space for rotational movement without damaging either element. This approach is realistic as a flush connection would cause undue damage to either element and prevent the device from moving and dissipating energy. The overall design approach is thus one of a hinge with a separate energy dissipation device.

\section{Performance Sensitivity to No-Slip Construction Requirements}

This section discusses the rigidity of the connection attachments and their effect on overall performance. The effect of increasing connection rigidity stiffness is presented in Fig. 6 . The initial tests for both configurations used a purely bolted connection. However, once testing commenced on for the case for two dampers located above the bottom flange of the beam, slippage was evident. Due to loss of dissipation from the device mounts slipping, greater

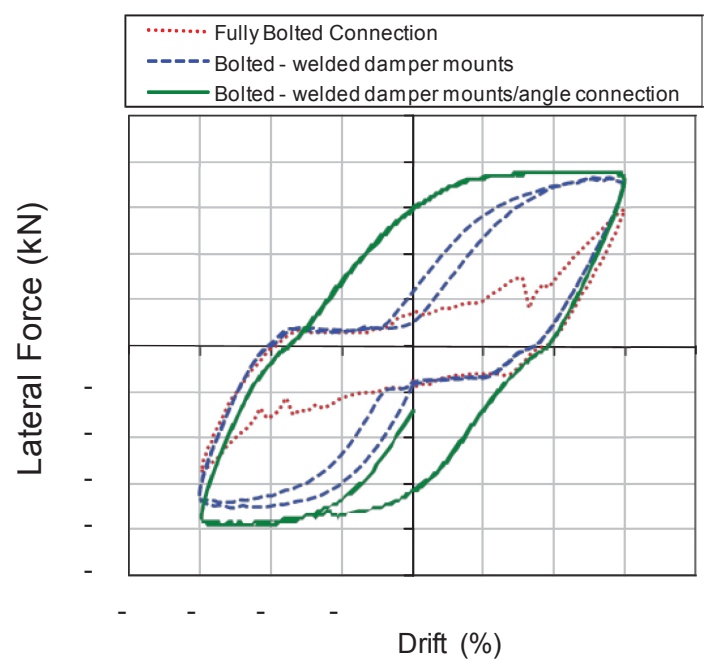

Fig. . Effect of bolted versus no-slip (welded) damper mounts onto bottom beam flange

by progressively welding up the attachment fixtures (angles) to inhibit slippage.

From a construction standpoint, the bolted connection is the preferred connection type, as it leads to increased construction speeds and eliminates another specialized on-site trade-welding. Bolted-only connections reduce costs and minimize the time for the required inspections and approvals.

Fig. 6 shows three levels of rigidity for the connection fixtures. The innermost lops within Fig. 6 show results for the fully bolted connection-the large amount of measured slip is attributed to the movement of the device mounts and the top angle. The overall hysteresis loops are pinched and minimal energy is dissipated as the bracket mounts slide. Sliding stopped when the bolts reached the end of their oversized holes at a drift of about $0.5 \%$. Note that all bolt holes were oversized by $2 \mathrm{~mm}$, as is customary practice.

After this slippage, the stiffness increases in Fig. 6 as any remaining slip occurs mostly from the top angle. The amount of energy dissipated at this stage was computed to be $1.61 \mathrm{~kJ}$.

After the damper mounts were tack welded to the beam (flange and web) this unintended movement was partially prevented and the hysteretic behavior was improved, as shown by the intermediate dashed (blue) line in Fig. 6. The energy dissipated was approximately doubled, increasing to $3.36 \mathrm{~kJ}$. However, some slip still occurred at low forces following a change in direction of applied force. This behavior was mitigated by welding a length of the end of the angle to the top beam flange to provide the required shear strength. The result produced a no-slip rigid angle-to-beam connection capable of dissipating energy to the capable limits of the HF2V dampers. The energy dissipated for the same $3 \%$ peak drift level was then $4.98 \mathrm{~kJ}$, in excess of three times greater than

the energy initially dissipated with a fully bolted connection.

restraint was provided to the device mounts. This was overcome 
Pekcan et al. (1999) introduced the concept of an energy ab- sorption efficiency factor, ' $\mathrm{fl}$, defined as the ratio of the area con- tained within a hysteresis loop, to an elastoplastic response with the same initial stiffness and strength. Thus, for the 3\% drift am- plitude shown in Fig. 6 the energy absorbed by one elastoper- fectly plastic loop is $5.76 \mathrm{~kJ}$. Based on this criteria, energy absorption efficiency factors of $30 \%, 58 \%$, and $86 \%$ are found for the fully bolted, partially welded, and full-welded bracket condi- tions, respectively. If all connections through out a frame structure were similar, then for the $3 \%$ drift amplitude this translates 
into respective equivalent viscous damping factors of $13 \%, 24 \%$, and 36\% (Pekcan et al. 1999).

According to previous work done by Pekcan et al. (1999) and Shama et al. (2002), the energy efficiency factor, ' $f l$, for steel structures is $60 \%$ (frames) and $75 \%$ (steel piles bent in good condition), respectively. Therefore, the fully welded rigid system exceeds a typical steel frame by a factor of 1.33 while the lessoptimal partly welded solution $' f l=58 \%$ ) matched the typical sacrificial design ' $\mathrm{fl}=60 \%$ ). It is thus evident that the use of well detailed HF2V devices incorporated directly into steel beam-tocolumn joint connections can provide superior energy dissipation than what is available using conventional welding techniques. These energy efficiency factors are a very simplified indication of how these connections could be incorporated into a performance-based design methodology. Previous research has looked at the addition of the HF2V devices on a single degreeof-freedom system (Rodgers et al. 2008), looking at the response spectra for suites of ground motion records. From this spectral investigation average acceleration reduction factors were determined, which can be used in capacity design methods (Rodgers et al. 2007,2008). A more extensive multidegree-of-freedom analysis will provide more extensive investigation of the influence of this design approach, and is the subject of ongoing research.

The use of two devices mounted above the flange revealed that for this specific design, the achievement of a fully bolted connection is not really feasible. Slip prevention criteria require twice the number of bolts used in the top angle. Providing the extra bolts is not viable due to the size of prefabricated steel angle available. However, a custom design bracket, prefabricated offsite in relatively large numbers required for a large structure would eliminate this issue and enable a purely bolted design.

A top angle bracket bolted into the column and beam flanges was used for the experimental protocol. In practice it would be desirable to have a bottom angle as well to seat the beam during erection (to act as a corbel) before fastening the top angle connection. This bottom angle would exist as a partial nonconnected seat, and could form part of the damper mount for the bottom mounted case. This angle may be considered for both construction purposes and as a fail-safe catcher seat in the unlikely event the umns elements. Repeatable energy dissipation on each cycle was observed with no strength deterioration, regardless of the magnitude of prior cycle. Compared to conventional steel frame connections a far greater dissipative efficiency of $86 \%$ (versus 60\%) was achieved without sacrificial damage.

3. The research investigated two locations for mounting the dampers: one damper below the bottom flange $j>1$ ); and two concealed dampers mounted above the bottom beam flange $j \quad 1$ ). In both cases it is essential to mount the damp- ers to ensure a no-slip condition of the mounting fixtures.

The more efficient design occurs when $j>1$, as this minimizes damper force and hence cost, as well as maximizing overall connection (and hence frame) stiffness.

4. For architectural considerations it may be desirable to conceal the dampers above the beam flange. For cases when $j$

1 , this may mean some welding of brackets to the beam is necessary to improve overall connection rigidity.

\section{References}

Christopoulos, C., Filiatrault, A., Uang, C.-M., and Folz, B. (2002). "Posttensioned energy dissipating connections for moment-resisting steel frames." J. Struct. Eng., 128(9), 1111-1120.

Christopoulos, C., Tremblay, R., Kim, H. J., and Lacerte, M. (2008) "Self-centering energy dissipative bracing system for the seismic resistance of structures: Development and validation." J. Struct. Eng., 134(1), 96-107.

Cousins, W. J., and Porritt, T. E. (1993). "Improvements to lead-extrusion damper technology." New Zealand Nat. Soc. Earthquake Eng. Bull, 26, 342-348.

Garlock, M. M., Ricles, J. M., and Sause, R. (2005). "Experimental studies of full-scale posttensioned steel connections." J. Struct. Eng., 131(3), 438-448.

Garlock, M. M., Sause, R., and Ricles, J. M. (2007). "Behavior and design of posttensioned steel frame systems.” J. Struct. Eng., 133(3), 389-399.

Mander, J. B., Chen S. S., and Pekcan, G. (1994). "Low-cycle fatigue behavior of semi-rigid top-and-seat angle connections." Eng. J., - 
=top angle fails due to low cycle fatigue. The top angle bracket is the only form of damage on the beam-column joint, and is easily 
Pekcan, G., Mander, J. B., and Chen, S. S. (1999). "Fundamental considreplaced by keeping the connection fully bolted, if need be. In seismic resistant structures the sacrificial bracket may be replaced if fatigued, which can be more easily done if an additional angle at the bottom flange of the beam exists to support the floor weight.

\section{Conclusions}

From the research presented in this paper the following conclusions are drawn:

1. The sacrificial damage that is expected to occur in conventional welded steel moment frame connections and hinge zones can be avoided by strategically reconfiguring the joint zone to include HF2V devices as the principal energy dissipating elements.

2. At $50 \%$ of full scale, a set of proof-of-concept experiments were undertaken to validate the proposed concept of adding dampers to a top flange-hung bolted connection. This design formed a near-rigid connection dissipating considerable energy at lateral drifts up to $4 \%$. Throughout the experiments there was no damage to the main structural beam and col- erations for the design of non-linear viscous dampers." Earthquake Eng. Struct. Dyn., 28, 1405-1425.

Pekcan, G., Mander, J. B., and Chen, S. S. (2000). "Experiments on steel MRF building with supplemental tendon system." J. Struct. Eng., 126(4), 437-444.

Ricles, J. M., Sause, R., Garlock, M. M., and Zhao, C. (2001). "Posttensioned seismic-resistant connections for steel frames." J. Struct. Eng., $127(2), 113-121$.

Robinson, W. H., and Greenbank, L. R. (1975). "Properties of an extrusion energy absorber." New Zealand Nat. Soc. Earthquake Eng. Bull, $8(3), 187-191$.

Robinson, W. H., and Greenbank, L. R. (1976). "An extrusion energy absorber suitable for the protection of structures during an earthquake." Earthquake Eng. Struct. Dyn., 4(3), 251-259.

Rodgers, G. W., Chase, J. G., Mander, J. B., Leach, N. C., and Denmead, C. S. (2007). "Experimental development, tradeoff analysis, and design implementation of high force-to-volume damping technology." New Zealand Nat. Soc. Earthquake Eng. Bull, 40(2), 35-48.

Rodgers, G. W., Mander, J. B., Chase, J. G., Dhakal, R. P., Leach, N. C., and Denmead, C. S. (2008). "Spectral analysis and design approach for high force-to-volume extrusion damper-based structural energy dissipation." Earthquake Eng. Struct. Dyn., 37(2), 207-223.

Shama, A. A., Mander, J. B., and Chen, S. S. (2002). "Seismic investigation of steel pile bents: II. Retrofit and vulnerability analysis." Earthquake Spectra, 18(1), 143-160. 
569 Skinner, R. I., Robinson, W. H., and McVerry, G. H. (1993). An intro570 duction to seismic isolation, Wiley, New York.

571 Solberg, K. M. (). "Experimental and financial investigations into

\#4 572 the further development of damage avoidance design." ME thesis, 573 Univ. of Canterbury, Christchurch, New Zealand.

\# 574 Solberg, K. M., Bradley, B. A., Rodgers, G. W., Mander, J. B., Dhakal, R. 575 P., and Chase, J. G. (). "Multi-level seismic performance assess- ment of a damage-protected beam-column joint with internal lead 576 dampers." Proc, New Zealand Society for Earthquake Engineering 577 Annual Conf. (NZSEE 2007), ., -

Solberg, K. M., Dhakal, R. P., Mander, J. B., Li, L., and Bradley, B. A. 579 A@

(). "Seismic performance of damage-protected beam column 580 joints." ACI Struct. 1., • , - 